\title{
Effects of Use and Disuse on Non-paralyzed and Paralyzed Skeletal Muscles
}

\author{
David R. Dolbow ${ }^{1 *}$ and Ashraf S. Gorgey ${ }^{2,3}$ \\ ${ }^{1}$ School of Kinesiology, College of Health, University of Southern Mississippi, Hattiesburg, MS \\ 39406, USA \\ ${ }^{2}$ Spinal Cord Injury and Disorders Service, Hunter Holmes McGuire VAMC, Richmond, VA 23249, \\ USA \\ ${ }^{3}$ Department of Physical Medicine and Rehabilitation, Virginia Commonwealth University, Richmond, \\ VA 23298, USA
}

[Received August 6, 2015; Revised August 26, 2015; Accepted August 26, 2015]

\begin{abstract}
Skeletal muscle is an integral part of the somatic nervous system and plays a primary role in the performance of physical activities. Because physical activity is vital to countering the effects of aging and age related diseases and is a key component in the maintenance of healthy body composition it is important to understand the effects of use and disuse on skeletal muscle. While voluntary muscle activity provides optimal benefits to muscle and the maintenance of healthy body composition, neuromuscular electrical stimulation may be a viable alternative activity for individuals with paralysis. Body composition with a healthy muscle to fat ratio has been associated with healthy blood lipid and glucose profiles that may decrease the risk of cardiovascular and metabolic diseases.
\end{abstract}

Key words: skeletal muscle, muscle paralysis, metabolic profile, electrical stimulation

Knowledge of skeletal muscle physiology is important as the various principles that govern muscle health guide the development of rehabilitative and preventive exercise prescriptions. This is especially true concerning the detrimental effects of aging and age related diseases. Skeletal muscle is a pliable tissue that depends on activity and responds differently according to the exercise stimulus. These include alterations in molecular, metabolic and functional properties [1] and can yield a spectrum of adaptations that range from muscle hypertrophy to fatigue resistance.

Conversely, skeletal muscle atrophy due to disuse or immobilization can have major consequences including decreased muscle fiber cross-sectional area (CSA) and reduced force generating capacity [2]. This process can lead to insulin insensitivity and subsequent type II diabetes with increased risk of cardiovascular diseases, thus it is important to explore countermeasures to ameliorate the effects of disuse on skeletal muscle. Voluntary muscle action is the natural order of muscle contraction however when unavailable, neuromuscular electrical stimulation (NMES) has been proposed as a promising rehabilitative tool for individuals with limited ability to voluntarily activate muscles.

The objectives of this paper are to 1) provide a brief overview of skeletal muscle strength, 2) describe the effects of muscle deactivation on muscle size, fiber type and muscle mechanics, 3) discuss the changes in body composition and metabolic profile accompanied with muscle atrophy, and 4) describe the effects of voluntary and externally induced exercise as countermeasures for reversing muscle disuse. 


\section{Muscle Strength and Specific Tension}

Skeletal muscle strength is a major component of maintaining mobility and activities of daily living. Moreover, skeletal muscle strength has long been tied to all-cause mortality [3]. Therefore, accurate estimation of muscle strength is necessary to understand possible deviations from the normal pattern. The maximum force normalized to the cross sectional area (CSA) or mass of the muscle has been considered as an accurate estimation of muscle strength [4-8]. This index is referred to as specific tension and accounts for differences in muscle size across different populations, ages (young vs. elderly) and gender [9-11] and neuromuscular factors of loss or gain in strength [9-11].

Several methods have been used to estimate the specific tension of muscle. Muscles of rodents have been stimulated directly and/or indirectly via the nerve, measuring the force and weighing the muscle [12]. Additionally, single motor units are stimulated to deplete glycogen and to estimate the CSA of the stimulated muscle fibers [1]. Muscle CSA has been measured in cadavers using indirect methods such as limb circumference corrected for subcutaneous fat $[9,14,15]$. Lastly, torque has been measured in humans with skeletal muscle size measured by non-invasive imaging techniques such as Doppler ultrasound, dual energy x-ray absorptiometry and magnetic resonance imaging (MRI) to calculate specific tension $[5,6,11]$.

Despite the use of MRI, there is a major methodological flaw in calculation of specific tension. This flaw is that no study has reported in vivo specific tension after accounting for the activated skeletal muscle size. It has been shown that muscle is not totally activated during maximum voluntary contraction and that muscle space is occupied by contractile and non-contractile tissue [16-18]. Therefore, failure to distinguish the difference between the two during measurement can result in overestimation of the amount of skeletal muscle engaged in torque production and underestimation of specific tension. Additionally, it questions previous results that used specific tension to compare between different aging groups (young vs. elderly) because activation differs with aging. For example, estimation of muscle recruited during exercise when referring to oxygen uptake/ unit muscle [19].

The activity of muscle contraction is the elemental key to muscle health and physical well-being. Following we explore the effects of muscle deactivation on muscle size, fiber type characteristics and muscle mechanics.

\section{Muscle Fiber Size and Deactivation}

Muscle size and strength are governed by the muscles capability to perform work. This concept can be divided into two simple principles known as the "Principle of Overload" and the "Principle of Reversibility". The Principle of Overload states that a greater than normal stress or load must be placed on the muscle to illicit a training response which includes muscle hypertrophy and increased work capacity [19]. Similarly, the Principle of Reversibility states that the amount of stress or load on a muscle must be sufficiently maintained in order to retain its size and work capacity [20]. If sufficient load or work is not maintained, muscle size and force capability will revert back to pre-adaptation size and work capacity levels. If work becomes absent, muscle atrophy continues unabated. This is typically thought to be a natural progression during aging as well as during chronic disorders such as cancer, chronic renal disease, human immunodeficiency virus, stroke and spinal cord injury (SCI).

While increased workload causes muscle hypertrophy or enlargement of muscle fibers, the lack of physical activity and insufficient load on muscles results in atrophy which is characterized by loss of muscle mass and muscle weakness. Decreased muscle size and strength typically have a linear relationship with physical inactivity. This fact is commonly realized with prolonged bed rest or immobilization due to casting and splinting. Early significant losses in muscle mass result from decreased protein synthesis which has been shown to occur after just 10 days of bed rest [21]. After the initial decreased levels of protein synthesis, muscle atrophy is continued by muscle protein breakdown [22]. This action is also intertwined with the aging process as typically people lose muscle mass as they age. However, individuals that are more physically active are able to retard the loss of muscle mass and maintain a vibrant level of physical functioning throughout life promoting the idea that decreased physical activity plays a more prominent role than chronological aging.

Muscle loss is perhaps most easily demonstrated by studying individuals that suffer an upper motor neuron injury resulting in some level of muscle paresis such as cerebral vascular or spinal cord injuries. People suffering from hemiparesis lose muscle mass and strength in the involved extremities without a decrement in function or strength of the intact extremities. Ryan and associates were able to measure an average $20 \%$ loss of muscle CSA coupled with a $25 \%$ increase in intra-muscular fat in the paretic mid-thigh of those with hemiparesis after stroke [23]. Likewise, 
individuals with incomplete SCI show muscle weakness and loss of muscle mass below the level of injury according to the severity of the injury.

After SCI, skeletal muscle atrophy appears to proceed at its highest rates within the first six weeks and reaches its nadir by the end of the first year. Those with complete motor SCI have the largest amount of muscle wasting as voluntary muscle activity becomes absent as opposed to merely decreased. Individuals that suffer a motor complete SCI can have extreme reductions in skeletal muscle CSA ranging from 45-80\% [22,24,25]. However, looks can be deceiving when observing the extremity sizes of those with SCI as intramuscular fat has been shown to increase as much as $126 \%$ in the first 6 weeks post SCI obscuring the visual evidence that muscle CSA has significantly decreased [25]. It has been reported that fat cells constitute up to $30 \%$ of muscle area in biopsied muscles after 8-10 years of denervation and that individuals with chronic SCI have three times more intramuscular fat and nearly four times more sub-fascial fat than people without SCI $[17,26]$. Decreased muscle mass is accompanied by narrowing of the membrane surrounding each muscle fiber (sarcolemma), decreased fiber size and decreased metabolism.

\section{Fiber Type Conversion}

While it is more accurate to describe skeletal muscle fiber types as a continuum based on histochemical and biochemical characteristics, [27] for simplicity of discussion, human skeletal muscle is typically divided into two main fiber types, slow oxidative twitch fibers (type I) and fast twitch fibers (type II) which is further divided into fast-glycolytic twitch fibers (type IIx) and fast-oxidative twitch fibers (type IIa). For further simplification these fibers may be thought of as three separate fiber types with slow-oxidative fibers and fastglycolytic fibers being on the opposite ends of the fiber type spectrum and fast-oxidative twitch fibers as the intermediate fiber [28]. The biochemical characteristics of the muscle fibers guide the contractile properties of each. Type I, slow-oxidative twitch fibers are so named because of the large number of mitochondria, oxidative enzymes and circulatory vessels in and around the muscle fiber [28]. These characteristics give this fiber type the ability to efficiently carry out aerobic metabolism producing adenosine triphosphate (ATP) for energy over an extended period of time. This process is highly fatigue resistant. Aerobic metabolism is a slower process relative to glycolytic metabolism. Type IIx, fast-glycolytic twitch fibers have very low numbers of mitochondria thus have a limited ability to carry out aerobic metabolism and are less resistant to fatigue compared to the type I oxidative twitch fibers. These fibers have a large number of glycolytic enzymes which makes them highly efficient in anaerobic energy metabolism [28]. Type IIa, fastoxidative twitch fibers have biochemical properties of both slow-oxidative and fast-glycolytic fibers. These fibers are unique in that they are more adaptable and with training can increase their oxidative or aerobic properties [29]. While some human muscles have higher amounts of particular muscle fiber types, there is typically a mixture of the three fibers types with the healthy human muscular system having an even mixture of fast and slow fiber types [29]. However, the relative body composition relationship between fast and slow fiber types has been shown to be variable due to heredity, race, sex and the characteristic plasticity of muscle fibers with training.

Just as muscle size is determined in large part by relative workloads during muscle contractions and physical activity levels, so too is the plasticity of muscle fiber types. Skeletal muscle is able to adapt to different forms of training by altering fiber type characteristics. The most common adaptation seen is the transformation of fast twitch fibers to slow twitch fibers by the increase in number of mitochondria and increased vascularization surrounding the muscle fibers. Interestingly, both endurance training and weight training have been shown to promote this same transformation although endurance training provides a much more efficient transformation [29]. Conversely, transition from slow twitch fibers to fast twitch fibers due to training although conceivable, is still an uncertainty owing to more evidence through research. Aging also affects the ratio and efficiency of fiber types with a transition from type IIx fast twitch fibers to type I slow twitch fibers. As with the loss of muscle mass with aging, this may be more descriptive of a decreased amount of physical activity and a change in the types of activities performed as people age than descriptive of an increased number of years of life.

Muscle denervation after stroke and SCI has also been shown to greatly affect the relative relationship between fast and slow twitch muscle fibers. While study results have varied there is a general consensus that a transition from slow to fast twitch fibers and an increase in fibers that show both fast and slow twitch properties arise after paralysis from stroke and SCI [30-33]. This transformation process was demonstrated early on by Grimby et al. as they found that several individuals from seven months to ten years post traumatic SCI were estimated to have approximately $90 \%$ fast twitch muscle fibers in vastus lateralis, gastrocnemius and soleus muscles [30]. Likewise, over 3 decades ago Landin and colleagues found that after CVA, there was a shift from slow twitch oxidative muscle fibers to fast twitch anaerobic based muscle fibers of the paretic vastus lateralis muscle [34]. Since then other researchers have 
discovered similar results in various paretic muscle groups $[32,35,36]$. This process typically shows manifestation between 4-7 months post injury and can continue up to 70 months post injury before plateauing into a new steady state of predominant type IIx fastglycolytic twitch muscle fibers [22]. The fact that muscle atrophy driven by upper motor neuron injuries show skeletal muscle shifts from slow oxidative twitch muscle fibers to fast glycolytic twitch muscle fibers and muscle atrophy related to the aging process shows a shift in the reverse direction may indicate that neurogenic properties are involved in the former.

\section{Mechanical Factors in Muscle Contraction}

There are several mechanical factors that play a key role in muscle function and training including the various functional contraction types, the muscle length at the time of contraction and the speed of contraction.

There are three primary functional muscle contraction types with three different purposes. The first is an isometric muscle contraction where the external force and the contraction force generated by the muscle are equal resulting in no movement of the body part completing the muscle contraction. This type of contraction is important when the intent of the activity is to hold an object in place i.e. holding a jog under a faucet to fill with water. The elbow flexor muscles must hold the elbow joint at a fixed angle to allow the water to flow into the opening of the jug. The second is a concentric muscle contraction during which the muscle exerts a greater force than the external object allowing movement of the object toward the body i.e. lifting the jug of water toward the mouth to drink. During this movement the elbow flexors must exert enough force to overcome the weight of the jug of water allowing the elbow to bend as the jug is moved toward the mouth. The third is eccentric muscle contraction whereby the force produced by the muscle lowers the external object allowing the object to move away from the body in a controlled fashion i.e. the weight of the jug is allowed to move away from the mouth by allowing the elbow flexors to slowly elongate in a controlled fashion as the elbow joint extends. Eccentric muscle contraction can actually produces more muscle force that isometric or concentric contractions [37]. Although each muscle contraction is vastly different they each play a vital role in body function.

The strength of a muscle's ability to contract is dependent upon its pre-contraction length, or degree of contraction. Levangie and Norkin discovered that a muscle can contract with maximal concentric force at 1.2 times its resting length. Beyond this length, active tension decreases due to insufficient sarcomere overlap [38].
Muscle contraction is also affected by the speed of muscle contraction and similar to muscle length, there is an optimal speed that allows the greatest force produced during muscle contractions. Because the force generated by muscle depends on the total number of actin-myosin cross-bridge connections, as the muscle contractions become faster there is less time for cross-bridge links to form decreasing the number and decreasing the force of the muscle contraction. With slower muscle contractions there is time for the optimal number of actin-myosin linkages to form thus maximizing force produced. Just as physical activity levels play a role in fiber type and force production, they also play a major role in body composition and metabolism.

\section{Body Composition and Metabolic Profile after Muscle Atrophy}

Muscle disuse due to inactivity can have a significant impact on body composition depending on the degree and length of disuse. When muscle is no longer activated to perform work, muscle atrophy follows. During the first several days of disuse muscle undergoes a slowing of protein synthesis which retards the body's ability to replenish contractile protein for muscle cells $[39,40]$. This process is followed by muscle protein breakdown (proteolysis) $[39,40]$. When proteolysis becomes greater than protein synthesis, muscle mass decreases resulting in altered body composition.

As stated earlier muscle disuse can result in dramatic losses in muscle mass. However, muscle disuse also plays a major role in the loss of bone mass and the accumulation of adipose tissue. Concerning bone, muscular activity is a primary contributor to bone health as muscle contractions cause the muscle tendon attached to bone to produce stress to the bone. This results in the cellular adaptation of bone increasing bone mineral density. This process follows the Mechanostat Theory, which was described by Frost as a derivative of Wolff's law which states that "there are strains within bone that are kept within certain limits by adding and removing bone tissue, which in turn strengthens the bone according to the particular stresses that are exerted upon it" $[41,42]$. These effects have been summarized along with the effects of SCI and exercise on bone health by Dolbow and associates [43].

The impact of skeletal muscle atrophy on body composition is clearly demonstrated in persons with SCI $[44,45]$. Deterioration in body composition and associated health issues following SCI has become a topic of interest among a growing number of investigators [46-50]. A few months post-injury, there is a rapid onset of skeletal muscle atrophy $[24,25,51]$ increase in fat mass (FM) $[45,46,50]$, waist circumference [49] and a decrease in fat free mass (FFM) [46,47]. Healthy body mass index (BMI) 
fails to reflect the percentage of FM after SCI as there is a significant gain in FM post-injury [52]. The deterioration in body composition and the associated muscle atrophy has been attributed to a number of factors including reduction in physical activity, unloading, disuse and reduction in anabolic hormone secretion [53,54]. The process of muscle atrophy begins within a few weeks of injury and continues at least until the end of the first year $[24,25]$. Skeletal muscle CSA can be decreased by more than half compared to healthy able-bodied (AB) controls $[22,24,51]$. Spungen et al. reported that monozygotic twins with acquired paraplegia had significantly more total body FM (7\%) and percent fat (13.1\%) per unit BMI than their AB twins [46]. Those with SCI showed an increase in $\mathrm{FM}(7 \%)$ compared with their $\mathrm{AB}$ co-twins. Spungen et al. has demonstrated that 133 men with SCI were $13.1 \%$ fatter per unit of BMI compared with age-, height-, and ethnicity-matched $\mathrm{AB}$ controls [47]. Individuals with SCI have greater trunk FM, visceral adipose tissue (VAT) CSA (58\%) and VAT: subcutaneous adipose tissue (SAT) ratio (48\%) compared to the age and waist circumference matched $\mathrm{AB}$ population [55-57]. The body composition changes may be further exacerbated by associated reductions in the anabolic hormones, testosterone ( $\mathrm{T})$, and growth hormone $(\mathrm{GH})$ and the $\mathrm{GH}$ secondary messenger insulin like growth factor-1 (IGF-1) [58,59]. GH release is blunted and chronically depressed after SCI, as evidenced by reduced levels of IGF-1, an indicator of chronic GH secretion [58,59]. In both rat and human models, reduction in IGF-1 has been associated with skeletal muscle atrophy and increases in FM [60]. Skeletal muscle atrophy and increased FM have been associated with decreased levels of IGF-I which is an indicator of GH secretion. GH plays a major role in promoting protein synthesis and muscle growth which also plays a role in controlling blood glucose levels and insulin sensitivity. Therefore, reductions in IGF-1 due to decreased muscle mass could partially explain increased levels of glucose intolerance and insulin resistance that are seen in individuals with stroke and SCI. In a recent study of 12 weeks of NMES and resistance training, IGF-1 increased by $25 \%$ in persons with motor complete SCI [61]. Moreover, increasing muscle activity via spasticity is capable of keeping the circulating plasma IGF-1 43\% higher in those with greater spasticity compared to those with less spastic skeletal muscle [62]. These findings warrant further study to explore the role of IGF-1 in response to disuse and exercise following SCI.

Reduced skeletal muscle activity and extreme muscle atrophy can lead to excessive accumulation of body fat. A healthy ratio of muscle to FM is accomplished by maintaining a balance between energy consumption and energy expenditure. This homeostatic balance is in large part accomplished through the combination of physical activity and the resting metabolic rate which are both largely governed by skeletal muscle mass. When the energy balance is disrupted by excessive increases in either energy consumption or decreased energy use additional adiposity results. Thus an active skeletal muscular system is important to maintain this balance. This process can again be clearly demonstrated by observing the consequences of SCI. After injury, the loss of metabolically active muscle mass results in reduction of the resting energy expenditure (EE). The resting EE accounts for $\sim 84 \%$ of the total daily (EE) thus significant decline in EE after SCI can dramatically disturb energy balance [53]. Using whole body potassium counts, Bauman et al. demonstrated a significant reduction in muscle mass in SCI compared to monozygotic $\mathrm{AB}$ twins $(2534 \pm 911$ vs. $3515 \pm 916)$. Bauman et al. also demonstrated that resting EE were similarly reduced in SCI compared to AB twins $(1634 \pm 290$ vs. $1735 \pm 295$ $\mathrm{kcal} / \mathrm{d}$ ) [63]. Without appropriate intervention, the decrease in resting metabolic rate and the decrease in physical activity overwhelm the energy balance and energy intake exceeds energy expenditure resulting in excessive adiposity.

As demonstrated in the above evidence, skeletal muscle is a key factor in maintaining healthy body composition. However, skeletal muscle activity and body composition have also been found to play a role in the maintenance of healthy metabolic blood profiles.

\section{Carbohydrate or Lipid Profiles and Muscle Atrophy}

Evidence has tied deterioration in skeletal muscle and the subsequent negative alteration in body composition to disorders of carbohydrate and lipid profile [64-67]. Duckworth et al. found an approximate $50 \%$ susceptibility rate among 45 participants being tested for problems with glucose intolerance, insulin resistance and type $2 \mathrm{DM}$ [68]. Bauman and Spungen documented the prevalence of type 2 diabetes mellitus was 3.5 times higher in persons with SCI (22\%) compared to AB controls (6\%). Fifty percentage of those with paraplegia and $82 \%$ with tetraplegia were diabetic [69-70]. In a study of persons with tetraplegia, Aksnes et al. similarly noted an association between whole body insulin-mediated glucose uptake and skeletal muscle mass suggesting that loss of muscle mass plays a primary role in insulin insensitivity [71]. It has also been suggested that the shift to fast twitch muscle fibers after stroke resulting in less fatigue resistant fibers could play a role in insulin resistance after stroke [1]. In addition to carbohydrate disorders, individuals with chronic physical disorders are characterized by abnormal atherogenic profiles because of dyslipidemia. Serum lipid levels in individuals with SCI showed a 
decrease in high-density lipoprotein cholesterol (HDL-C) that negatively correlated with an increase in serum triglycerides (TG) [72,73]. Nash et al. reported that 76\% of individuals with paraplegia had HDL-C less than 40 $\mathrm{mg} / \mathrm{dl}$ and 34\% had the Adult Treatment Panel III- defined metabolic syndrome [66]. The above complications have been strongly correlated to a reduction in physical activity and deterioration in body composition (increase body adiposity and reduction in FFM) that predispose individuals with SCI to a spectrum of health-related secondary complications. Likewise, Nelson and associates reported that $55 \%$ of individuals with SCI were at risk of developing metabolic syndrome [67]. It is also possible that increases in FM are associated with inflammatory biomarkers that trigger metabolic syndrome after SCI. Adipose tissue has been demonstrated to secrete large amounts of pro-inflammatory cytokines, including interleukin-6 (IL-6) and tumor-necrosis factor- $\alpha$ (TNF$\alpha$ ). This stimulates hepatic production of $\mathrm{C}$ - reactive protein (CRP) which is tied to vascular inflammation [74].

Advances in imaging technology allow successful determination of the relationships between body composition adaptations and metabolic profile after SCI. Several studies have revealed the association between excessive adipose tissue accumulation and altered metabolic profile. In a cross-sectional study, Gorgey and Gater established associations between regional or relative adiposity and metabolic dysfunction after SCI. The study showed that in individuals with SCI, leg FM had higher metabolic associations to carbohydrate and lipid profiles compared to whole body FM, especially when leg FM was adjusted to whole body FM [75]. The findings show that interventions that decrease leg FM may reverse the metabolic disorders after SCI. Whole body FM is negatively related to HDL-C concentration after controlling for percentage trunk FM [75]. An advantage of using magnetic resonance imaging over dual-energy $\mathrm{X}$ ray absorptiometry is the capability to differentiate between subcutaneous adipose tissue and ectopic adipose tissue. Edwards et al. noted significant association between VAT, plasma insulin and insulin resistance index; while a negative correlation was noted between VAT: SAT ratio and HDL-C. Increases in VAT has also been shown to be related to leptin and plasminogen activator inhibitor-1 [56,57]. Percent whole body FFM was negatively related to VAT and SAT volumes [65], suggesting that strategies that increase lean mass would be associated with reduction in central adiposity and can attenuate the deleterious effects of ectopic adipose tissue on the metabolic profile of individuals with SCI. The findings also confirm observations that exercise induced skeletal muscle hypertrophy is associated with reduction in ectopic adipose tissue [76].
The evidence of association between skeletal muscle loss and unfavorable metabolic profiles continues to increase, however further corroborative research is needed before a clear cause-effect relationship can be substantiated. Regardless of this substantiation, it is clear that skeletal muscle disuse can have devastating consequences for sedentary individuals. However, in many cases these effects can be reversed through increased physical activity.

\section{Exercise as a Countermeasure to Skeletal Muscle Disuse}

Exercise is commonly prescribed as an effective therapeutic modality to induce a healthy musculoskeletal system, reduce obesity and enhance metabolic profile in the AB community. The Centers for Disease Control and the American College of Sports Medicine recommendations have indicated that daily exercise for 30 minutes or more is required to maintain health and reduce the risk of sedentary life-style associated diseases [77,78]. However, for those with physical disabilities several barriers exist limiting participation in beneficial regular physical activities. For example after SCI, exercise opportunities are either not possible or limited to small muscle mass above the level of lesion which does not utilize the large muscle groups in the lower extremities [79]. This renders exercise as an ineffective method of attenuating the deterioration in body composition and metabolic profile after SCI.

Since the primary cause of skeletal muscle atrophy is muscle disuse, it makes sense that the primary treatment for muscle atrophy is muscle use. For $\mathrm{AB}$ persons that have lost muscle mass due to sedentary life-style, a change to a more physically active life-style is typically all that is required. However, for individuals that have been immobilized for weeks i.e. casting or splinting, a rehabilitation program guided by healthcare professionals is in order. Berg and colleagues have shown that unilateral casting for 4 weeks resulted in $7 \%$ reduction in the CSA as well as $22 \%$ decrease in torque of the calf muscle [80]. Jones and associates induced skeletal muscle atrophy on healthy individuals by immobilizing a leg for two weeks followed by a 6 week rehabilitation exercise program. After 2 weeks of immobilization, force production was decreased accompanied by the increase of proteolytic genes and a near $5 \%$ decrease in skeletal muscle mass. After 6 weeks of rehabilitation exercise, these factors reversed [81].

\section{Electrical Stimulation as a Countermeasure for Muscle Atrophy}


For individuals with chronic muscle paralysis, exercise options are limited. Volitional exercises such as armcrank, hand-rim wheelchair propulsion, and hand cycling are generally used for endurance training, while resistance exercises are used for upper body strength training [82]. However, none of these activities resolve the problem of skeletal muscle loss in paralyzed limbs. Functional electrical stimulation lower extremities cycling (FESLEC) using surface electrodes (Figure 1) has become a primary alternative for those with paralysis and has a long history of safe use in rehabilitation centers evoking contractions in paralyzed muscles [83]. Table 1 highlights the benefits of FES-LEC on body composition which have been shown to include increased muscle mass, decreased fat mass and increased bone mineral density. Griffin et al. also reported improvements in blood glucose and insulin sensitivity [84-91].

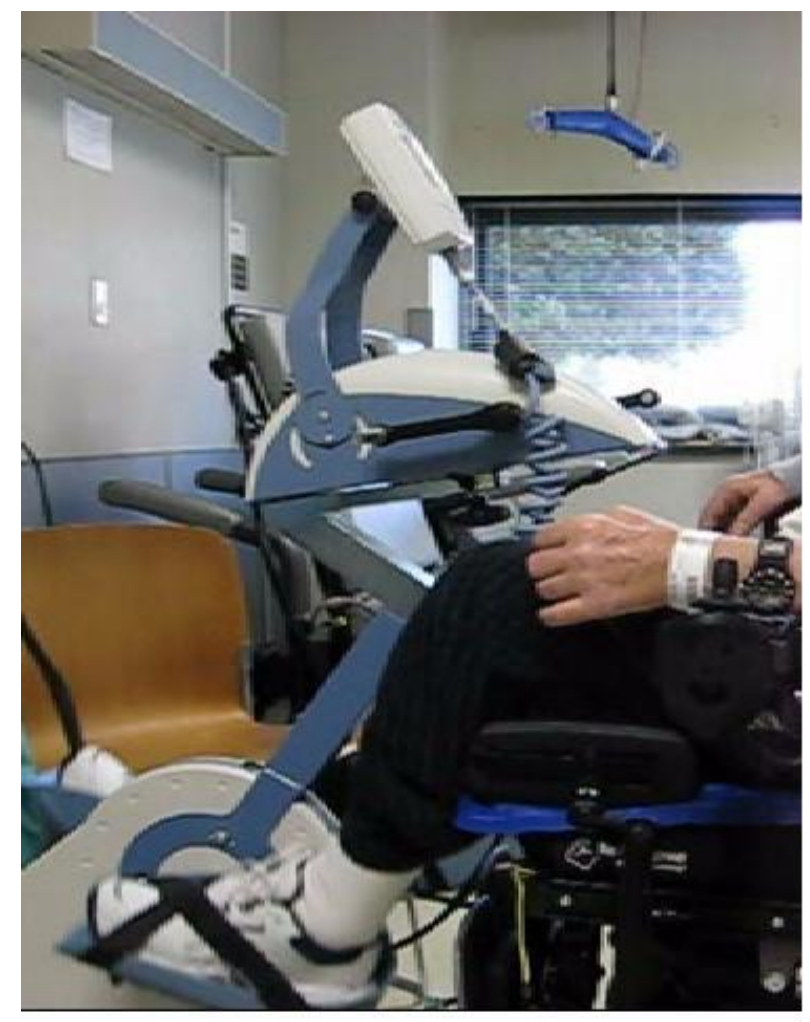

Figure 1. Functional Electrical Stimulation Lower Extremity Cycling. Restorative therapies (RTI) powered FES bike that has been successfully applied for home exercise program in persons with spinal cord injury. The progress of the training as well as adjustment of the FES parameters can be monitored via and controlled via internet.

Mahoney et al. showed that 12 weeks of evoked resistance training (RT) using surface NMES resulted in
$40 \%$ hypertrophy of the knee extensor muscle group with a trend of improvement in glucose tolerance in individuals with motor complete SCI [92]. Gorgey et al. found that electrical stimulation activities that evoked leg extension exercise combined with diet (RT+ diet group) outperformed diet control (diet group) alone. Using the technology of MRI (Figure 2), the findings detected skeletal muscle hypertrophy. Skeletal muscle hypertrophy of the whole thigh (28\%) and knee extensor (35\%) muscle groups were observed in the RT+ diet group with no changes in the diet group. Other muscle groups (knee flexors) showed significant hypertrophy (12-16\%) in the RT + diet group. Skeletal muscle hypertrophy was associated with an increase in plasma IGF-1 (25\%) in the $\mathrm{RT}+$ diet group but not in the diet group. The increase in IGF-1 was negatively associated with ectopic adipose tissue accumulation (\%IMF and VAT). The outcomes of 12 weeks of RT also suggested that \%leg FM (decreased by $6 \%$ ) and was associated with an increase in absolute leg FFM by $10 \%$ in the RT+ diet compared to no changes in the diet group. The results showed that there is a trend of VAT reduction in the RT+ diet group with a $25 \%$ decrease of VAT in the region of L5-S1 to S2-S3. The RT +diet showed a favorable change on the carbohydrate and lipid profiles compared to diet only. Plasma insulin area under the curve (AUC) decreased in the RT + diet ($34 \%)$ group but not in the diet $(+22 \%)$ group, with no change in plasma glucose. Plasma TG $(-37 \%$ vs. $+12.6 \%)$ and cholesterol: HDL-C $(6 \pm 2$ to $5 \pm 1$ vs. $5 \pm 1$ to $5 \pm 1)$ decreased and HDL-C (10\%) increased in the RT+ diet group compared to the diet group [61]. The findings suggested that evoking skeletal muscle hypertrophy could be simply achieved by gradually lifting ankle weights for twice weekly without the need for engaging in a long-term exercise on a daily basis. Most important is that the outcomes of training was accompanied with improvement in both carbohydrate and lipid profiles. It should be noted that it is advisable to monitor caloric intake and regulate factors such as pain and stress during exercise [61]. In a report that involves FES-LEC for 21 weeks, 4 times per week where caloric intake was not monitored; training was unsuccessful in inducing positive body composition or metabolic adaptations in a person with SCI [93]. Therefore, monitoring caloric intake is highly recommended when designing training studies that aim to induce body composition or metabolic adaptations.

\section{Electrical Stimulation and Muscle Fatigue}

Applications of surface NMES can cause premature onset skeletal muscle fatigue that limits the outcomes of exercise in individuals with physical disabilities [94]. Investigators have focused their effort into studying the effects of NMES parameters and skeletal muscle 
recruitment in studying the rapid onset of muscle fatigue during NMES. For example, increasing the frequency and duty cycle from $10 \mathrm{~Hz}$ and $50 \%$ to $50 \mathrm{~Hz}$ and $70 \%$ causes $60 \%$ fatigue for the later compared to only $20 \%$ for the former [95]. Gorgey et al. introduced 4 protocols of surface NMES to the knee extensor muscle group to study the independent effects of frequency, amplitude of the current and pulse duration on muscle fatigue [96]. The first protocol was set to deliver $100 \mathrm{~Hz}$, amplitude of the current that required to evoke $75 \%$ of the maximum voluntary contraction (MVC) and pulse duration of 450 $\mu \mathrm{s}$. The three other protocols were modified from the first protocol by changing only one parameter and the rest of the parameters were kept constant. In the $2^{\text {nd }}$ protocol, pulse duration was altered from $450 \mu \mathrm{s}$ to $150 \mu \mathrm{s}$, the third protocol frequency was reduced from 100 to $25 \mathrm{~Hz}$ and finally in the fourth protocol, the amplitude of the current was reduced from $75 \%$ to $45 \%$ of the current required to evoke MVC. The results showed that altering the amplitude of the current or pulse duration did not influence muscle fatigue; whereas reducing the frequency from $100 \mathrm{~Hz}$ to $25 \mathrm{~Hz}$ reduces the percentage decline in force by $37 \%$ [96]. It is well known electrically stimulated training with surface NMES reduces muscle fatigue. Sabatier et al. studied the effects of electrically stimulated training following 12 weeks of resistance training in men with complete SCI. The findings showed that there was $60 \%$ reduction in muscle fatigue [97]. However, the authors showed that reduction in muscle fatigue was not associated with improvement in arterial diameter or blood flow capacity [97].

Table 1. Benefits of FES-LEC on Body Composition

\begin{tabular}{|c|c|c|c|}
\hline Authors & Times/Wk & $\begin{array}{l}\text { Program } \\
\text { Duration }\end{array}$ & Results \\
\hline Skold et al. 2002 [84] & $2-3 / \mathrm{wk}$ & 6 months & $10 \%$ increase in muscle mass \\
\hline $\begin{array}{l}\text { Hjeltnes et al. } 1997 \\
{[85]}\end{array}$ & $7 \mathrm{x} / \mathrm{wk}$ & 8 weeks & $\begin{array}{l}2 \% \text { increase in muscle mass, } 22 \% \text { increase in muscle CSA, } 1.9 \% \\
\text { decrease in fat mass }\end{array}$ \\
\hline Griffin et al. 2009 [86] & $3 \mathrm{x} / \mathrm{wk}$ & 10 weeks & $3.3 \%$ increase in muscle mass \\
\hline $\begin{array}{l}\text { Demchak et al. } 2005 \\
\text { [87] }\end{array}$ & $3 \mathrm{x} / \mathrm{wk}$ & 13 weeks & $63 \%$ increase in muscle cross sectional area \\
\hline Mohr et al. 1997 [88] & $3 \mathrm{x} / \mathrm{wk}$ & 1 year & Increased bone mineral density in the tibia. \\
\hline Dolbow et al.2012 [89] & $3 \mathrm{x} / \mathrm{wk}$ & 9 weeks & $8.3 \%$ increase in lean mass, $1.2 \%$ decrease in fat mass. \\
\hline Dolbow et al. 2012 [90] & $3 \mathrm{x} / \mathrm{wk}$ & 6 months & $3.3 \%$ increase in lean mass, $7.1 \%$ increase in legs lean mass \\
\hline Dolbow et al. 2014 [91] & $3 \mathrm{x} / \mathrm{wk}$ & 1 year & $\begin{array}{l}7.7 \% \text { increase in lean mass, } 4.1 \% \text { increase in legs lean mass, } 1.2 \% \\
\text { decrease in fat mass, } 9.9 \% \text { decrease in legs fat mass. }\end{array}$ \\
\hline
\end{tabular}

Other studies have investigated the effects of recruitment on skeletal muscle fatigue during NMES. Compared to the size principle in which recruitment gradually progresses from the order of small slow twitch to large fast twitch motor units, recruitment of motor units during NMES does not necessarily follow the same principle. Recruitment during NMES has been an active area of research investigation and may explain the rapid onset of premature fatigue. Initially and based of the work of muscle biopsy, the order of recruitment was thought to be the reverse of the size principle where large fast twitch fibers are recruited before slow twitch fibers. Because large twitch fibers rely primarily on glycolysis, these fibers are unable to replenish their glycogen storage capacity during NMES and hence the idea of reversal recruitment. Another theory describes a randomly, synchronous, repeated recruitment of motor units during NMES compared to the orderly, asynchronous recruitment during voluntary exercise (i.e. slow to fast fibers as force is increased) [98]. In addition, the fatigued motor units can potentially be compensated for by the recruitment of additional units during voluntary effort as fatigue ensues in an effort to maintain sub-maximal performance [99]. In contrast, with NMES repeated random activation of the same slow and fast motor units result in fatigue during submaximal exercise so performance cannot be maintained. The process of muscle fatigue during NMES is well documented in persons with physical disabilities. Persons with SCI were unable to show recovery in muscle torque between repeated bouts of electrical stimulation compared to $\mathrm{AB}$ controls. The study clearly documented that persons with SCI had $66 \%$ decline of the knee extensor torque compared to $33 \%$ for AB controls [100]. NMES applied with large pulse duration $1 \mathrm{~ms}$ can result in volley like effect and stimulate the alpha motor units via the afferent ant-dromic pathway. Collins and associates have conducted a series of studies that have determined the capabilities of surface NMES to cause asynchronous recruitment similar to volitional exercise via afferent stimulation [101]. The work showed 
that there is a volley-like phenomenon that may result in afferent stimulation and indirectly stimulate the motor neuron pool to activate the desired muscle group [101]. This work may potentially reduce the rapid muscle fatigue during stimulation. The same group also tested the difference in recruitment between the direct stimulation of the tibial nerve and the calf muscle. Direct stimulation of the tibial nerve resulted in more asynchronous recruitment compared to stimulation of the calf muscle which resulted in the typical random, motor unit recruitment. The authors suggested that direct nerve stimulation should be considered as a technique to minimize muscle fatigue during NMES [102]. However, the clinical applicability of this technique may be site specific and may be difficult to be used for stimulation of the femoral nerve to activate the knee extensor muscle group, especially in obese individuals.
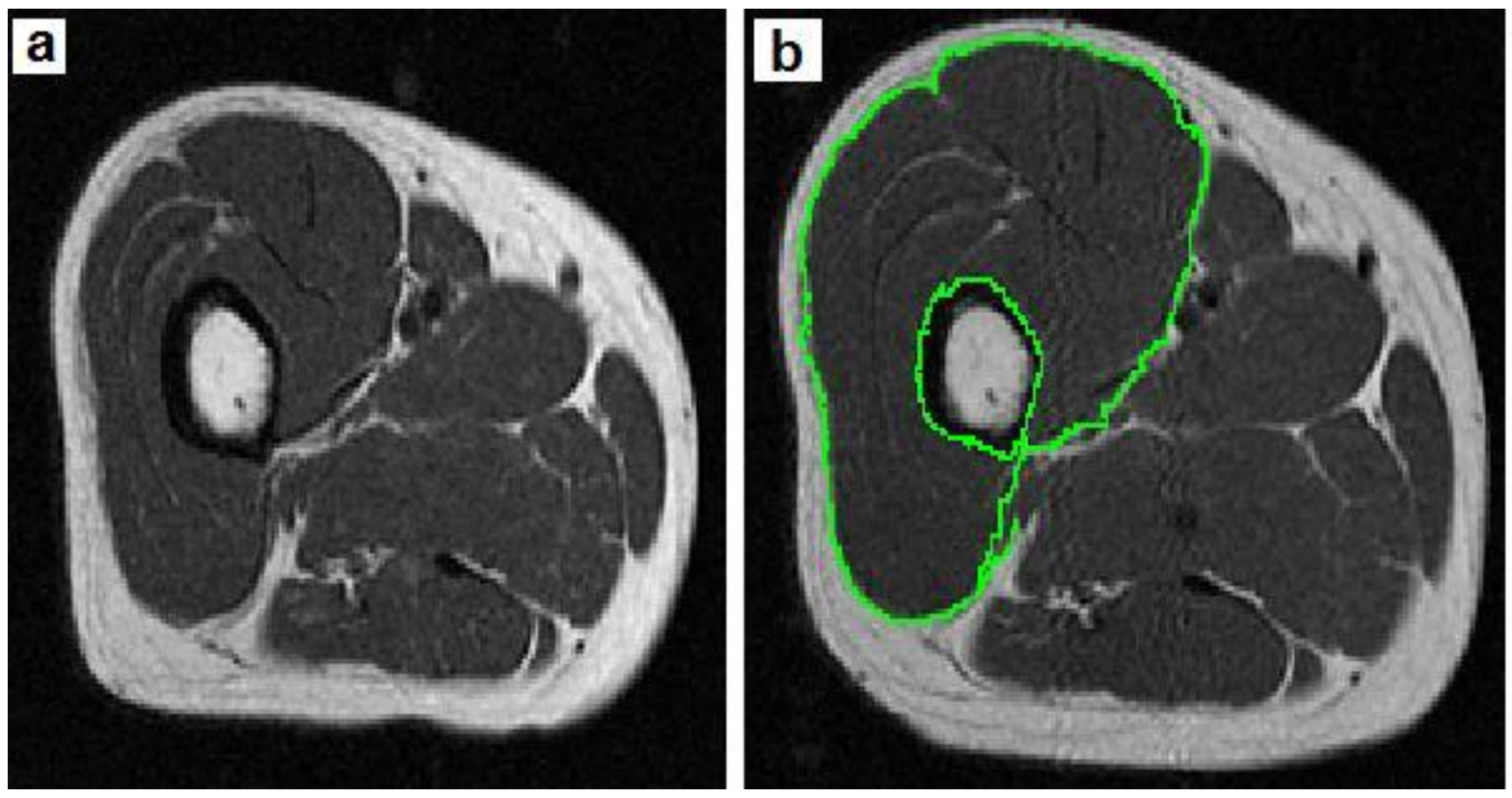

Figure 2. Pre- and Post- Training Mid-thigh Imaging. A representative T1 MRI images of the mid-thigh in a person with motor complete SCI A) pre and B) post-12 weeks of training using neuromuscular electrical stimulation to the knee extensor muscle group to evoke dynamic leg extension exercise while lifting ankle weights.

Although it is clear that FES activities are able to increase muscle mass, the effect on bone is not clear. Groah and associates found that electrical stimulation of the quadriceps muscle 5 times per week for 12 weeks resulted in a significant slowing of the bone loss with 26 individuals in the early stages of SCI [103]. Studying chronic SCI adults, Frotzler and colleagues found significant increases in total BMD and regional BMD in the distal femur after FES-LEC 3 to 4 times per week for 6 and 12 months [104]. Mohr studied BMD of the proximal tibia in 10 persons with chronic C6-T4 SCI with FES-LEC for 30 minutes 3 times per week for 12 months followed by 6 months of once weekly FES-LEC [88]. Prior to FES-LEC the SCI group showed 52\% less BMD in the proximal tibia than that of the control group. After 12 months of FES-LEC performed 3 times per week, BMD had increased by $10 \%$. However, after 6 months of once weekly FES-LEC, the difference in BMD between the SCI group and the control group had returned to 50\%, leading the authors to conclude that 3 times per week FES-LEC is beneficial for BMD however, once weekly FES-LEC does not maintain BMD in the proximal tibia [88]. Similarly, Chen and associates found that 6 months of FES-LEC improved the BMD of the distal femur and proximal tibia however, 6 months after cessation of FESLEC BMD returned to pre-exercise levels [105]. These findings demonstrate that the benefits of physical activity are temporary and that long term physical activity is required to achieve long term benefits.

\section{Conclusion}

Skeletal muscle health is linked to overall body health and both are linked to adequate physical activity. When 
physical activity is significantly decreased via central nervous system injury, alternative methods of producing muscle contractions must be utilized. Currently electrical stimulation is the only viable method of producing muscle contractions without stimulus from the higher centers of the central nervous system. Further study is needed to advance the technologies of these devices and to explore other possibilities.

\section{Declaration of Interest}

The authors have compiled with the guidelines for authorship and publishing set forth by the journal of Aging and Disease. The authors report no conflict of interest financial or otherwise. To the author's knowledge, all human and animal studies discussed within this manuscript were performed in accordance with the ethical standards laid down in the 1964 Declaration of Helsinki and later amendments.

\section{References}

[1] Hafer-Macko CE, Ryan AS, Ivey FM, Macko RF (2008). Skeletal muscle changes after hemiparetic stroke and potential beneficial effects of exercise intervention strategies. J of Rehabil Research \& Development, 45:261-272.

[2] Gorgey AS, Dolbow DR, Dolbow JD, Gater DR (2014). Effects of Spinal Cord Injury on Body Composition and Metabolic Profile-Part I. Journal of Spinal Cord Medicine, 37: 693-702.

[3] FitzGerald SJ, Barlow CE, Kampert JB, Morrow JR Jr, Jackson AW, Blair SN (2004). Muscular Fitness and All-Cause Mortality: Prospective Observations. Journal of Physical Activity and Health, 11:7-18.

[4] Edgerton VR, Roy RR. Regulation of skeletal muscle fiber size, shape and function (1991). J Biomech, 24 Suppl 1;123-133.

[5] Narici MV, Roi GS, Landoni L (1988). Force of knee extensor and flexor muscles and cross-sectional area determined by nuclear magnetic resonance imaging. Eur J Appl Physiol Occup Physiol, 57:39-44.

[6] Narici MV, Landoni L, Minetti AE (1992). Assessment of human knee extensor muscles stress from in vivo physiological cross-sectional area and strength measurements. Eur J Appl Physiol Occup Physiol, 65:438-444.

[7] Fukunaga T, Roy RR, Shellock FG, Hodgson JA, Edgerton VR (1996). Specific tension of human plantar flexors and dorsiflexors. J Appl Physiol, 80:158-165.

[8] Maganaris CN, Baltzopoulos V, Ball D, Sargeant AJ (2001). In vivo specific tension of human skeletal muscle. J Appl Physiol, 90:865-872.

[9] Buchanan TS. Evidence that maximum muscle stress is not a constant: differences in specific tension in elbow flexors and extensors (1995). Med Eng Phys, 17:529536.
[10] Grimby G, Aniansson A, Hedberg M, Henning GB, Grangard U, Kvist H (1992). Training can improve muscle strength and endurance in 78- to 84-yr-old men. J Appl Physiol, 73:2517-2523.

[11] Reeves ND, Narici MV, Maganaris CN (2004). Effect of resistance training on skeletal muscle-specific force in elderly humans. J Appl Physiol, 96:885-892.

[12] Cope TC, Bodine SC, Fournier M and Edgerton VR (1986). Soleus motor units in chronic spinal transected cats: physiological and morphological alterations. J Neurophysiol, 55:1202-1220.

[13] Bodine SC, Roy RR, Eldred E and Edgerton VR (1987). Maximal force as a function of anatomical features of motor units in the cat tibialis anterior. J Neurophysiol, 57:1730-1745.

[14] Wickiewicz TL, Roy RR, Powell PL and Edgerton VR (1983). Muscle architecture of the human lower limb. Clin Orthop Relat. Res, 275-283.

[15] Cureton KJ, Collins MA, Hill DW and McElhannon FM, Jr (1988). Muscle hypertrophy in men and women. Med Sci Sports Exerc, 20:338-344.

[16] Kandarian SC, White TP (1989). Force deficit during the onset of muscle hypertrophy. J Appl Physiol, 67:2600-2607.

[17] Elder CP, Apple DF, Bickel CS, Meyer RA, Dudley Ga (2004). Intramuscular fat and glucose tolerance after spinal cord injury--a cross-sectional study. Spinal Cord, 42(12):711-6.

[18] Kendall TL, Black CD, Elder CP, Gorgey A, Dudley GA (2006). Determining the extent of neural activation during maximal effort. Med Sci Sports Exerc, 38:14705.

[19] Nieman DC (2007). Exercise prescription in Exercise Testing and Prescription. Sixth Edition, McGraw Hill, Boston, MA. 230-281.

[20] Harris BA, Watkins MP. Adaptations to strength training in Exercise in Rehabilitation Medicine. Second Edition. 2006. Ed Frontera WR, Slovik DM, Dawson DM. Human Kinetics, Champaign, IL. 24-33.

[21] Kortebein P, Ferrando A, Lombeida J, Wolfe R, Evans WJ (2007). Effect of 10 Days of Bed Rest on Skeletal Muscle in Healthy Older Adults JAMA, 297:17721774.

[22] Biering-Sorensen BO, Kristenson IB, Kjaer M, BieringSorensen F (2009). Muscle after Spinal Cord Injury. Muscle \& Nerve, 40:499-519.

[23] Ryan AS, Dobrovolny CL, Smith GV, Silver KH, Macko RF (2002). Hemiparetic muscle atrophy and increased intramuscular fat in stroke patients. Arch Phys Med Rehabil, 83(12):1703-1707.

[24] Castro MJ, Apple DF, Jr. Hillegass EA, Dudley GA (1999). Influence of complete spinal cord injury on skeletal muscle cross-sectional area within the first 6 months of injury. Eur J Appl Physiol Occup Physiol, 80(4):373-378.

[25] Gorgey AS, Dudley GA (2007). Skeletal muscle atrophy and increased intramuscular fat after incomplete spinal cord injury. Spinal Cord, 45:304-309.

[26] Kern H, Boncompagni S, Rossini K, Mayr W, Fano G, Zanin ME, et al. (2004). Long term denervation in 
humans causes degeneration of both contractile and excitation-contraction coupling apparatus, which is reversible by functional electrical stimulation (FES): a role for myofiber regeneration? J Neuropathol Exp Neurol, 63:919-931.

[27] Ingalls CP (2004). Nature vs. nurture: can exercise really alter fiber type composition in human skeletal muscle. Journal of Applied Physiology, Nov. 97:15911592.

[28] Powers SK, Howley ET. Exercise Physiology: Theory and application to fitness and performance. $7^{\text {th }}$ Edition. McGraw Hill, Boston MA 2008; 154-165.

[29] Yan Z, Okutsu M, Akhtar YN, Lira VA (2011). Regulation of exercvise-induced fiber type transformation, mitochondrial biogenesis, and angiogenesis in skeletal muscle. Journal of Applied Physiology, 110:264-274.

[30] Grimby G, Broberg C, Krotkiewska I, Krotkiewski M (1976). Muscle fiber composition in patients with traumatic cord lesion. Scand J Rehabil Med, 8:37-42.

[31] Burnham R, Martin T, Stein R, Bell G, MacLean I, Steadward R (1997). Skeletal muscle fiber type transformation following spinal cord injury. Spinal Cord, 35:86-91.

[32] DeDeyne PG, Hafer-Macko CE, Ivey FM, Ryan AS, Macko RF (2004). Muscle molecular phenotype after stroke is associated with gait speed. Muscle Nerve, 30:209-215.

[33] Talmadge RJ, Castro MJ, Apple DF Jr, Dudley GA (2002). Phenotypic adaptations in human muscle fibers 6 and 24 wk after spinal cord injury. J Appl Physiol, 92:147-54.

[34] Landin S, Hagenfeldt L, Saltin B, Wahren J (1977). Muscle metabolism during exercise in hemipaetic patients. Clin Sci Mol Med, 53:257-269.

[35] Huey KA, Haddad F, Qin AX, Baldwin KM (2003). Transcriptional regulation of the type I myosin heavy chain gene in denervated rate soleus. Am J Physio Cell Physiol, 284:C738-748.

[36] Shields RK, Dudley-Javoroski S (2006). Musculoskeletal plasticity after acute spinal cord injury. Effects of long-term neuromuscular electrical stimulation training. J Neurophysiol, 95:2380-2390.

[37] Lindstedt SL, LaStayo PC, Reich TE (2001). When active muscles lengthen: Properties and consequences of eccentric contractions. News Physiological Science, 16:256-261.

[38] Levangie PK, Norkin CC (2005). Joint Structure and Function: A Comprehensive Analysis. Fouth Edition. F.A. Davis Company; 4 edition.

[39] Booth FW, Criswell DS (1997). Molecular events underlying skeletal muscle atrophy and the development of effective countermeasures. International Journal of sports Medicine, 18:S265-269.

[40] Powers SK, Smuder AJ, Judge AR (2012). Oxidative stress and disuse muscle atrophy: cause or consequence? Curr Opin Clin Nutr Metab Care, 15:2405.

[41] Frost HM (1987). "Mass" and the "mechanostat": a proposal. Anat Rec, 219:1-9.
[42] Frost HM (2003). Bone's mechanostat: a 2003 update. Anat Rec A Discov Mol Cell Evol Biol, 275:10811101.

[43] Dolbow DR, Gorgey AS, Daniels JA, Adler RA, Moore JR, Gater DR Jr (2011). The effects of spinal cord injury and exercise on bone mass: a literature review. NeuroRehabilitation, 29:261-9.

[44] Kocina P (1997). Body composition of spinal cord injured adults. Sports Medicine, 23:48-60.

[45] Gater DR (2007). Obesity after Spinal Cord Injury. Physical Medicine and Rehabilitation Clinics of North America, 18:331-351.

[46] Spungen AM, Wang J, Pierson RN, Bauman WA (2000). Soft tissue body composition differences in monozygotic twins discordant for spinal cord injury. Journal of Applied Physiology, 88:1310-1315.

[47] Spungen AM, Adkins RH, Stewart CA, et al. (2003). Factors influencing body composition in persons with spinal cord injury: a cross-sectional study. Journal of Applied Physiology, 95:2398-2407.

[48] Clasey JL, Gater DR, Jr (2005). A comparison of hydrostatic weighing and air displacement plethysmography in adults with spinal cord injury. Arch Phys Med Rehabil, 86:2106-2113.

[49] Buchholz AC, Bugaresti JM (2005). A review of body mass index and waist circumference as markers of obesity and coronary heart disease risk in persons with chronic spinal cord injury. Spinal Cord, 43:513-8.

[50] Gorgey AS, Chiodo AE, Zemper ED, Hornyak JE, Rodriguez GM, Gater DR (2010). Relationship of spasticity to soft tissue body composition and the metabolic profile in persons with chronic motor complete spinal cord injury. J Spinal Cord Med, 33:615.

[51] Dudley GA, Castro MJ, Rogers S, Apple DF Jr (1999). A simple means of increasing muscle size after spinal cord injury: a pilot study. Eur J Appl Physiol Occup Physiol, 80:394-6.

[52] Jones LM, Legge M, Goulding A (2003). Healthy body mass index values often underestimate body fat in men with spinal cord injury. Arch Phys Med Rehabil, 84:1068-71.

[53] Buchholz AC, McGillivray CF, Pencharz PB (2003). Physical activity levels are low in free-living adults with chronic paraplegia. Obes Res, 11:563-70.

[54] Tsitouras PD, Zhong YG, Spungen AM, Bauman WA (1995). Serum testosterone and growth hormone/insulin-like growth factor-I in adults with spinal cord injury. Horm Metab Res, 27:287-92.

[55] Mojtahedi MC, Valentine RJ, Arngrimsson SA, Wilund KR, Evans EM (2008). The association between regional body composition and metabolic outcomes in athletes with spinal cord injury. Spinal Cord, 46:192197.

[56] Maruyama Y, Mizuguchi M, Yaginuma T, Kusaka M, Yoshida H, Yokoyama K, Kasahara Y, Hosoya T (2008). Serum leptin, abdominal obesity and the metabolic syndrome in individuals with chronic spinal cord injury. Spinal Cord, 46:494-49. 
[57] Edwards LA, Bugaresti JM, Buchholz AC (2008). Visceral adipose tissue and the ratio of visceral to subcutaneous adipose tissue are greater in adults with than in those without spinal cord injury, despite matching waist circumferences. Am J Clin Nutr, 87:600-607.

[58] Bauman WA, Spungen AM, Flanagan S, Zhong YG, Alexander LR, Tsitouras PD (1994). Blunted growth hormone response to intravenous arginine in subjects with a spinal cord injury. Horm Metab Res, 26:152-6.

[59] Bauman WA, Kirshblum SC, Morrison NG, Cirnigliaro CM, Zhang RL, Spungen AM (2006). Effect of lowdose baclofen administration on plasma insulin-like growth factor-I in persons with spinal cord injury. J Clin Pharmacol, 46:476-82.

[60] Florini JR, Ewton DZ, Coolican SA (1996). Growth hormone and the insulin-like growth factor system in myogenesis. Endocr Rev, 17:481-517.

[61] Gorgey AS, Mather KJ, Cupp HR, Gater DR (2012). Effects of resistance training on adiposity and metabolism after spinal cord injury. Med Sci Sports Exerc, 44:165-174.

[62] Gorgey AS, Gater DR (2012). Insulin growth factors may explain relationship between spasticity and skeletal muscle size in men with spinal cord injury. J. Rehabil Res Dev, 49:373-80.

[63] Bauman WA, Spungen AM, Wang J, Pierson RN Jr (2004). The relationship between energy expenditure and lean tissue in monozygotic twins discordant for spinal cord injury. Rehabil Res Dev, 41:1-8.

[64] Duckworth WC, Solomon SS, Jallepalli P, Heckemeyer C, Finnern J, Powers A (1980). Glucose intolerance due to insulin resistance in patients with spinal cord injuries. Diabetes, 29:906-10.

[65] Gorgey AS, Mather KJ, Poarch H, Gater DR (2011). Influence of motor complete spinal cord injury on visceral and subcutaneous adipose tissue measured by multiaxial magnetic resonance imaging. J Spinal Cord Med, 34:99-109.

[66] Nash MS, Mendez AJ (2007). A guideline-driven assessment of need for cardiovascular disease risk intervention in persons with chronic paraplegia. Arch Phys Med Rehabil, 88:751-7.

[67] Nelson MD, Widman LM, Abresch RT, Stanhope K, Havel PJ, Styne DM, McDonald CM (2007). Metabolic syndrome in adolescents with spinal cord dysfunction. J Spinal Cord Med, 30Suppl1:S127-39.

[68] Duckworth WC, Jallepalli P, Solomon SS (1983). Glucose intolerance in spinal cord injury. Arch Phys Med Rehabil, 64:107-110.

[69] Bauman WA, Spungen AM (1994). Disorder of carbohydrate and lipid metabolism in veterans with paraplegia or quadriplegia: A model of premature aging. Metabolism, 43:749- 756.

[70] Bauman WA, Spungen AM (2001). Carbohydrate and lipid metabolism in chronic spinal cord injury. J Spinal Cord Med, 24:266-277.

[71] Aksnes AK, Hjeltnes N, Wahlström EO, Katz A, Zierath JR, Wallberg-Henriksson H (1996). Intact glucose transport in morphologically altered denervated skeletal muscle from quadriplegic patients. Am J Physiol, 271:E593-600.

[72] Bauman WA, Spungen AM, Zhong YG, Rothstein JL, Petry C, Gordon SK (1992). Depressed serum high density lipoprotein cholesterol levels in veterans with spinal cord injury. Paraplegia, 30:697-703.

[73] Bauman WA, Spungen AM (2008). Coronary heart disease in individuals with spinal cord injury: assessment of risk factors. Spinal Cord, 46:466-476.

[74] Manns PJ, McCubbin JA, Williams DP (2005). Fitness, inflammation, and the metabolic syndrome in men with paraplegia. Arch Phys Med Rehabil, 86:1176-81.

[75] Gorgey AS, Gater DR (2011). Regional and Relative Adiposity Patterns in Relation to Carbohydrate and Lipid Metabolism in Men with Spinal Cord Injury. Appl Physiol Nutr Metab, 36:107-14.

[76] Gorgey AS, Mather KJ, Gater DR (2011). Central adiposity associations to carbohydrate and lipid metabolism in individuals with complete motor spinal cord injury. Metabolism, 60:843-51.

[77] Kraemer WJ, Adams K, Cafarelli E, Dudley GA, Dooly C, Feigenbaum MS, et al. (2002). American College of Sports Medicine position stand. Progression models in resistance training for healthy adults. Med Sci Sports Exerc, 34:364-80.

[78] Haskell WL, Lee IM, Pate RR, Powell KE, Blair SN, Franklin BA et al. (2007). Physical activity and public health: updated recommendation for adults from the American College of Sports Medicine and the American Heart Association. Med Sci Sports Exerc, 39:1423-34.

[79] Jacobs PL, Nash MS (2004). Exercise recommendations for individuals with spinal cord injury. Sports Med, 34:727-51.

[80] Berg HE, Dudley GA, Häggmark T, Ohlsén H, Tesch PA (1991). Effects of lower limb unloading on skeletal muscle mass and function in humans. J Appl Physiol, 70:1882-5.

[81] Jones SW, Hill RJ, Krasney PA, O’Conner B, Peirce N, Greenhaff PL (2004). Disuse atrophy and exercise rehabilitation in humans profoundly affects the expression of genes associated with regulation of skeletal muscle mass. FASEB J, 18:1025-1027.

[82] Valent, L. J. M., Dallmeijer, A. J., Houdijk, H., Slootman, H. J., Janssen, T. W., Post, M. W. M., and L. H. van der Woude (2009). Effects of Hand Cycle Training on Physical Capacity in Individuals with Tetraplegia: A Clinical Trial. Phys Ther, 89:1051-1060.

[83] Robinison CJ, Kett NA, Bolam JM (1988). Spasticity in Spinal-Cord Injured Patients .1. Short-Term Effects of Surface Electrical-Stimulation. Arch Phys Med Rehab, 69:598-604.

[84] Skold C, Lonn L, Harms-Ringdahl K, Hultling C, Levi R, Nash M, et al. (2002). Effects of functional electrical stimulation training for six months on body composition and spasticity in motor complete tetraplegic spinal cord-injured individuals. J Rehabil Med, 34(suppl 1):25-32.

[85] Hjeltnes N, Aksnes AK, Birkeland KI, Johansen J, Lannem A, Wallberg-Henriksson H (1997). Improved 
body composition after 8 wk of electrically stimulated leg cycling in tetraplegic patients. Am J Physiol, 273(suppl 3, pt 2):R1072-R1079.

[86] Griffin L, Decker MJ, Hwang JY, Wang B, Kitchen K, Ding Z, Ivy JL (2009). Functional electrical stimulation cycling improves body composition, metabolic and neural factors in persons with spinal cord injury. $\mathrm{J}$ Electromyogr Kinesiol, 19:614-22.

[87] Demchak TJ, Linderman JK, Mysiw WJ, Jackson R, Suun J, Devor ST (2005). Effects od functional electrical stimulation cycle ergometry training on lower limb musculature in acute SCI individuals. Journal of Sports Science \& Medicine, 4:263-271.

[88] Mohr T, Podenplant J, Biering-Sorenen F, Galbo H, Thamsborg G, Kjaer M (1997). Increased bone mineral density after prolonged electrically induced cycle training of paralyzed limbs in spinal cord injured man. Calcified Tissue International, 61:22-25.

[89] Dolbow DR, Gorgey AS, Cifu DX, Moore JR, Gater DR (2012). Feasibility of home-based functional electrical stimulation cycling. Spinal Cord, 50:170-1.

[90] Dolbow DR, Gorgey AS, Moore JM, Gator DR (2012). A Report of Practicability of a Six Month Home Based Functional Electrical Stimulation Cycling Program for an Individual with Tetraplegia. J Spinal Cord Medicine, 35:182-186.

[91] Dolbow DR, Gorgey AS, Gater DR, Moore JR (2014). Body composition after 12 months of FES cycling: case report of a 60-year-old female with tetraplegia. Spinal Cord, June;52(Suppl 1):S3-4.

[92] Mahoney ET, Bickel CS, Elder C, Black C, Slade JM, Apple Jr. D and Dudley GA (2005). Changes in skeletal muscle size and glucose tolerance with electrically stimulated resistance training in subjects with chronic SCI. Arch Phys Med Rehabil, 86:1502-4.

[93] Gorgey AS, Harnish C, Daniels J, Dolbow DR, Keeley A, Moore J, Gater DR (2012). A report of anticipated benefits of functional electrical stimulation after spinal cord injury. J Spinal Cord Medicine, 35:107-112.

[94] Binder-Macleod SA, Snyder-Mackler L (1993). Muscle fatigue: clinical implications for fatigue assessment and neuromuscular electrical stimulation. Phys Ther, 73:902-910.
[95] Lieber RL, Kelly MJ (1993). Torque history of electrically stimulated human quadriceps: implications for stimulation therapy. J Orthop Res, 11:131-141.

[96] Gorgey AS, Black CD, Elder CP, Dudley GA (2009). Effects of electrical stimulation parameters on fatigue in skeletal muscle. J Orthop Sports Phys Ther, 39:68492.

[97] Sabatier MJ, Stoner L, Mahoney ET, Black C, Elder C, Dudley GA, McCully K (2006). Electrically stimulated resistance training in SCI individuals increases muscle fatigue resistance but not femoral artery size or blood flow. Spinal Cord, 44:227-33.

[98] Knaflitz M, Merletti R and De Luca CJ (1990). Inference of motor unit recruitment order in voluntary and electrically elicited contractions. J Appl Physiol, 68:1657-1667.

[99] Maton B and Gamet D (1989). The fatigability of two agonistic muscles in human isometric voluntary submaximal contraction: an EMG study. II. Motor unit firing rate and recruitment. Eur J Appl Physiol Occup Physiol, 58:369-374.

[100] Bickel CS, Slade JM and Dudley GA (2004). Longterm spinal cord injury increases susceptibility to isometric contraction-induced muscle injury. Eur $\mathrm{J}$ Appl Physiol, 91:308-313.

[101] Collins DF (2007). Central contributions to contractions evoked by tetanic neuromuscular electrical stimulation. Exerc Sport Sci Rev, 35:102-9.

[102] Bergquist AJ, Clair JM, Lagerquist O, Mang CS, Okuma Y, Collins DF (2011). Neuromuscular electrical stimulation: implications of the electrically evoked sensory volley. Eur J Appl Physiol, 111:2409-26.

[103] Groah SL, Lichy AM, Libin AV, Ljungberg I (2010). Intensive electrical stimulation attenuates femoral bone loss in acute spinal cord injury. PMR, 2:11080-1087.

[104] Frotzler A, Coupland S, Perret C, Kakebeeke TH, Hunt KJ, Donaldson Nde N, et al. (2008). High Volume FES cycling partially reverses bone loss in people with chronic spinal cord injury. Bone, 43:169-176.

[105] Chen SC, Lai CH, Chan WP, Huang MH, Tsai HW, Chen JJ (2005). Increases in bone mineral density after functional electric stimulation cycling exercises in spinal cord injured patients. Disability \& Rehabilitation, 27:1337-1341. 